\title{
Comparative Investigation of Split Spectrum Processing over Classical Approach of Filtering for Non-Linear \& Non-Stationary Signals
}

\author{
S. Muthumari \\ AP-I, School of EEE, \\ SASTRA University, \\ Thanjavur-613401.
}

\author{
K.Vijayarekha \\ Professor, School of EEE, \\ SASTRA University, \\ Thanjavur-613401,
}

\author{
Amrit Chatterjee \\ SASTRA University, \\ Thanjavur-613401
}

\begin{abstract}
Ultrasonic welding defect signals are not processed, they are very difficult to identify the pattern of the defect, especially the TOFD welding defect signals are often mixed with noise and lead to confusion between grain noise and small flaws. It is very difficult to interpret the result defect and noise due to larger grain noise. This paper describes the comparative study of matlab simulation results of classic approach signal processing methodology and Split Spectrum Processing (SSP) with the Polarity Thresholding algorithm (PT) to the TOFD welding defect. A-scan signals are used to enhance SNR while suppressing the grain noise. The simulation results show that the welding defect TOFD signals after the SSP investigation are the best and produce the qualified output.
\end{abstract}

\section{Categories}

Experimental Setup, Split Spectrum Processing Technique, Simulation Results.

\section{Keywords}

Split spectrum processing (SSP), Time of Flight Diffraction (TOFD), Signal to Noise Ratio (SNR), Non Destructive Evaluation (NDE), Low pass filter.

\section{INTRODUCTION}

For detection and characterization of defects, various signal processing techniques are already used. The detection method quality may be estimated by the signal to noise ratio receipt system output [2][11] .Ultrasonic welding defect signals are nonlinear non-stationary signals[10]. In this paper structure, the signals are processed by conventional approach of signal processing methodology and compare the results to the Split spectrum processing which process the ultrasonic pulse echo system to identify the flaw echo from the ultrasonic noisy pulse signal [1].

Literature survey does not indicate specific work in the area of suppressing noise (SNR Enhancement) of welding defect TOFD signals using split spectrum processing technique. The SSP technique for TOFD welding defect signals with polarity thresholding algorithm which will produce effective SNR enhancement and clear waveforms namely lateral wave, defect diffracted wave and back-wall echo. If the signal is noise free, the defects are easily characterized[9]. Typical pattern of waves involved in TOFD technique is lateral wave, diffracted waves and back-wall echo [3][6].In Conventional filtering method, the noise component of the signal should be identified and given as input to this method so that we can get a qualified output. For the stationary signal, which don't vary much over time, classical approach of filtering network is suitable for suppressing noise. In this research we compare the SSP technique and classical approach of filtering technique for non-linear non-stationary TOFD welding defect signals.

\section{Experimental Setup:}

TOFD experiment model microplux of $\mathrm{M} \mid \mathrm{S}$ AEA technology, U.K with manual scanner along with longitudinal wave $(4 \mathrm{MHz})$ angle beam probe of $45^{\circ}$ (model WSY 45) was used for the experiment. A manual weld scanner was used is acquisition of several A-scans obtained by scanning of welded bead. After the inspection of 11 test samples a total of 330 signals obtained. The time taken to scan a length of weld is very short since no raster scanning is necessary. Probe separation should be maintained [4] between transmitter probe and receiver probe which is shown in experimental setup shown in fig 1 .

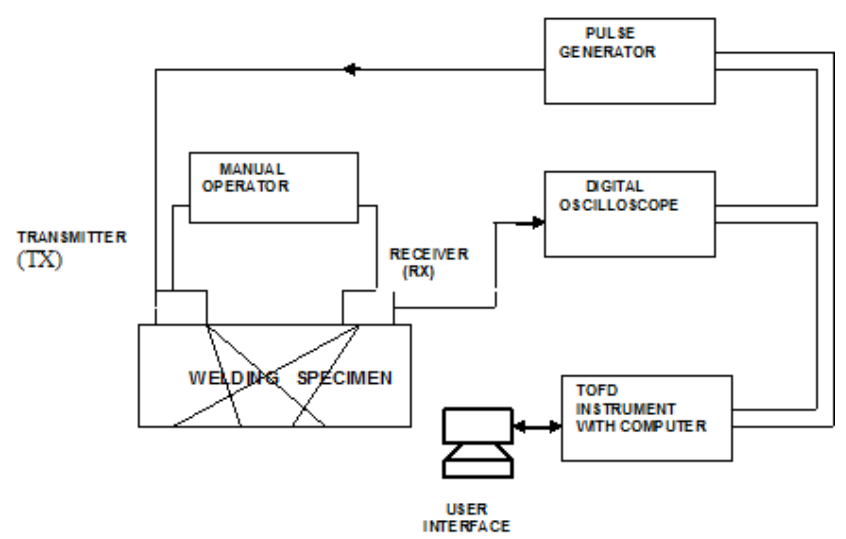

Figure 1: Experimental setup for getting TOFD welding defect signals.

\section{Split Spectrum Processing Technique:}

The SSP technique splits the spectrum of the received TOFD testing signal into different overlapping frequency bands by means of equally spaced band pass filters mentioned in fig2. The sub-band signals are then processed by applying a non-linear selection technique that retains signal information only when there is a strong correlation among most of the sub-bands at a given time instant. After getting the time domain signal Gn (t), we 
applied the polarity thresholding algorithm. In PT algorithm, when the flaw signal is present, the corresponding SSP data has same polarity, such that the flaw signal dominates the grain noise at least locally.

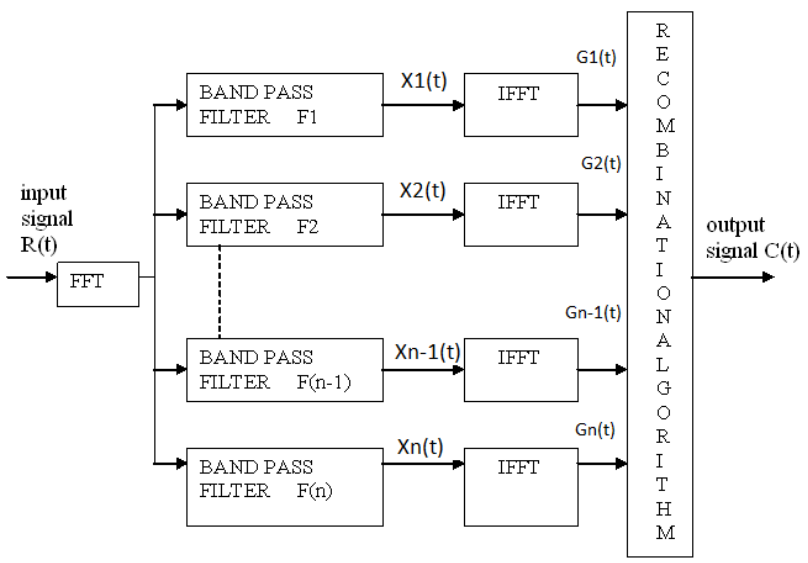

Figure 2: Split Spectrum Processing Technique

\subsection{Analysis}

SSP involves in decomposing the wideband signal into a number of smaller bands, the purpose being to separate the noise components which are not correlated from the actual flaw components which are highly correlated[8].The band in which the flaw lies along with the back-wall echo, surface wave as well as the noise from the larger grains. This dominant band must be split into smaller sub-bands in the next stage. The numbers of filters used are 4.The final design parameter is choosing recombination algorithm to be applied in reconstruction stage.

$$
\begin{array}{rlr}
\mathrm{C}(\mathrm{t}) & =\min \left|\mathrm{G}_{\mathrm{n}}(\mathrm{t})\right| \text {; if all } \mathrm{G}_{\mathrm{n}}(\mathrm{t})>0 & \mathrm{n}=0,1,2,3 \ldots \ldots \mathrm{k} \\
& =\min \left|\mathrm{G}_{\mathrm{n}}(\mathrm{t})\right| ; \text { if all } \mathrm{G}_{\mathrm{n}}(\mathrm{t})<0 & \mathrm{n}=0,1,2,3 \ldots \ldots \mathrm{k} \\
& =0 &
\end{array}
$$

\section{Simulation Results 4.1 SNR Enhancement}

All the 330 signals were taken from AISI steel plates having dimension $260 / 260 / 25 \mathrm{~mm}$. The results are shown for the signal named as LF-01. Fig 3(a) shows typical welding defect A- scan signal which possess the information lateral wave, defect diffracted wave and back-wall echo. TOFD welding flaw signal embed with highly scattering grain noise. The magnified version of high frequency component (due to scattering) present in the signal shown in fig $3(b)$.

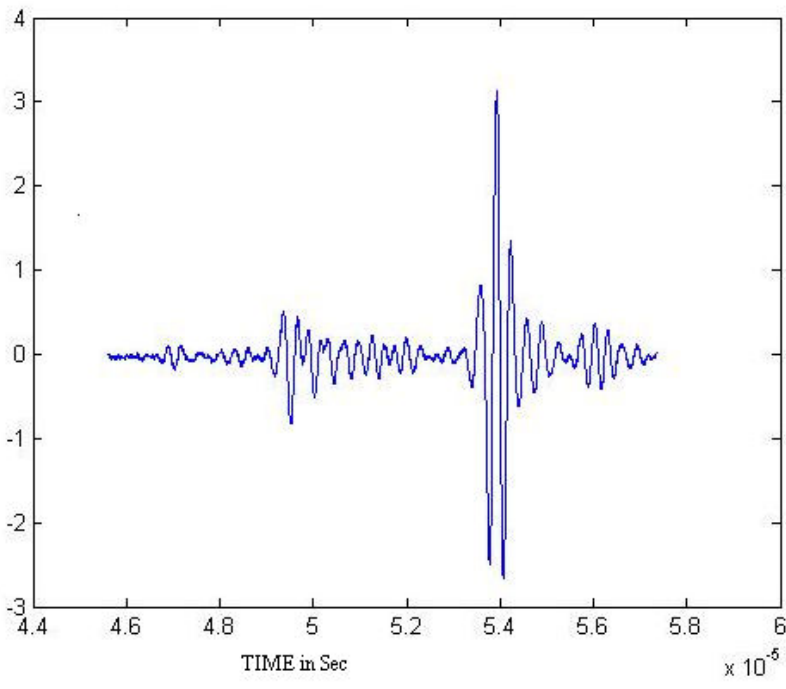

Figure 3(a): TOFD welding defect A-scan signal

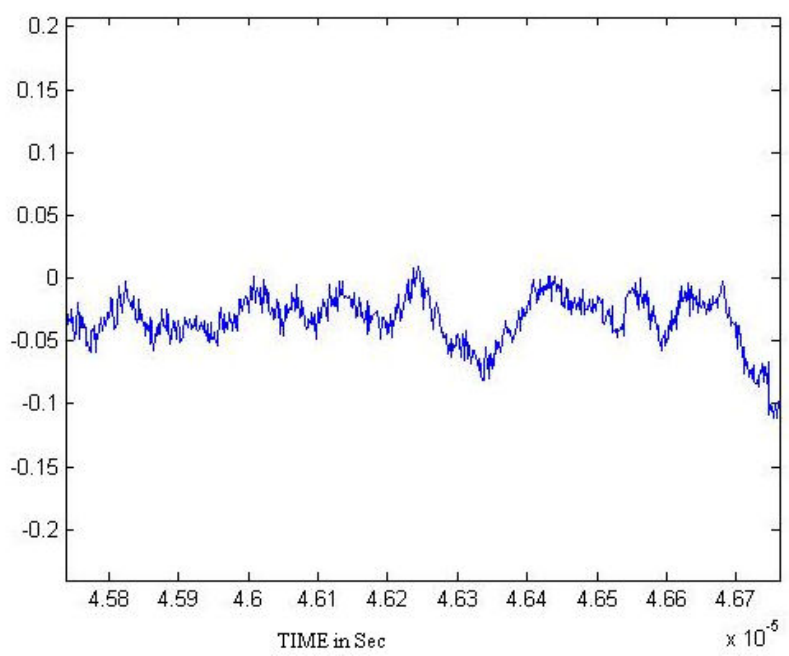

Figure 3(b): Magnified version of high frequency component present in TOFD welding defect signal.

When the signals are processed by classical approach of filtering, the noise component due to scattering cannot be removed completely. Using conventional filtering approach the noise is removed in TOFD welding defect signals is shown in fig 4 . Whilst all high frequency components are filtered, the signal doesn't give any qualified information about flaw. The TOFD welding defect signal is applied to SSP filtering network and its output is shown in fig6, where we can easily identify the defect location and backwall echo of the signal. Fig 5 shows the signal which is mixed with white Gaussian noise. Instead, for obtaining a qualified output we use SSP technique which is shown in fig6. Finally, table 1 gives the SNR enhancement data of 9 randomly selected signals from original batch of 330 signals. 


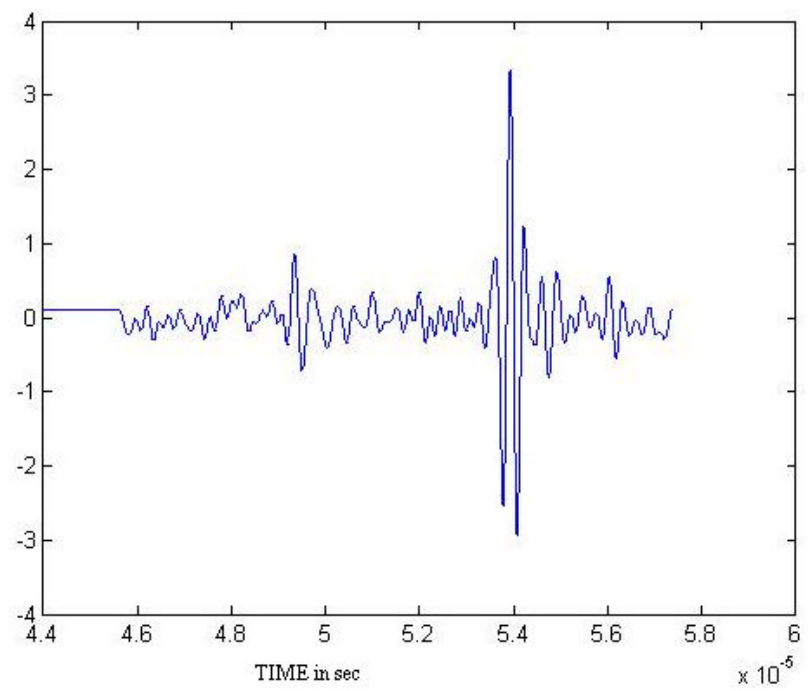

Figure 4:Classical Approach of filtering signals (LPF)

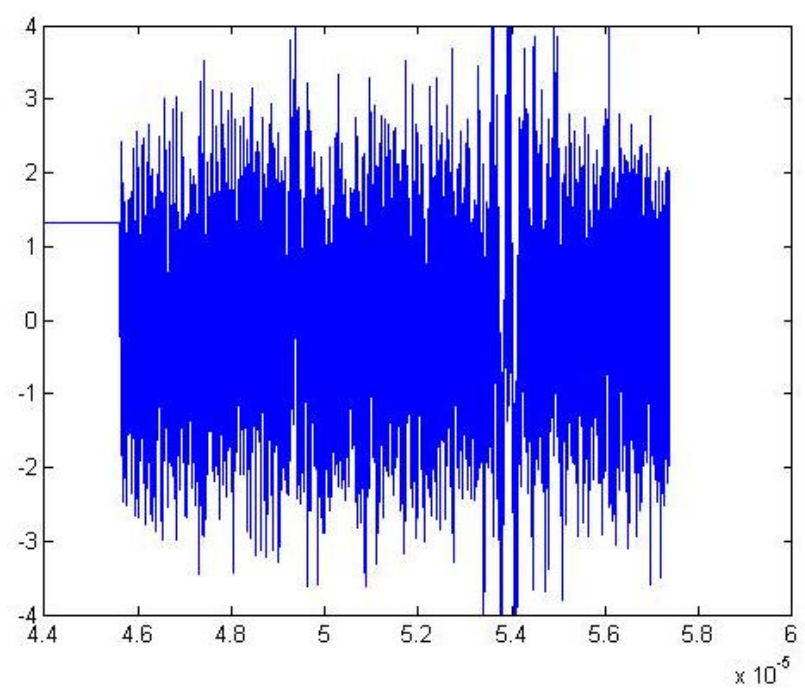

Figure 5:Signal mixed with white Gaussian noise

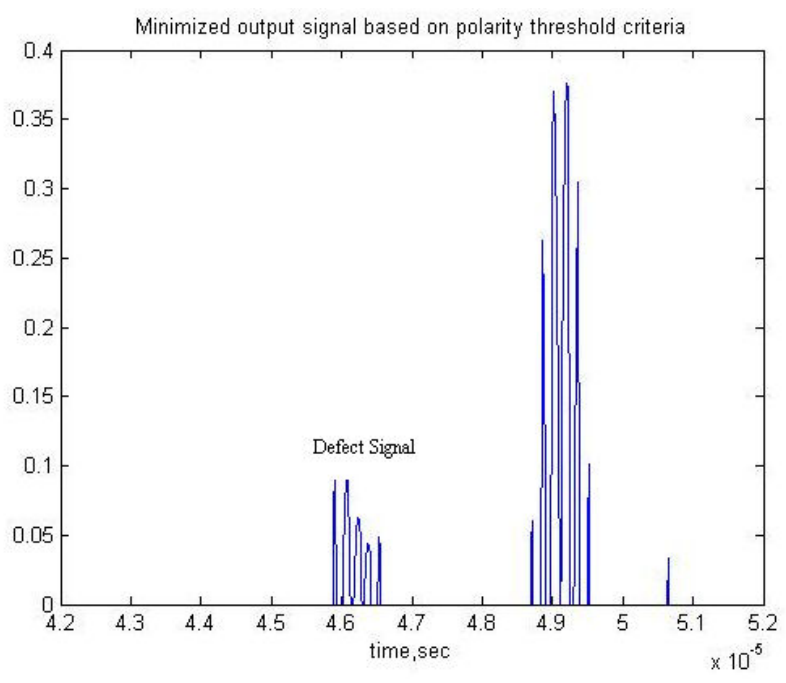

Figure 6: SSP filtered output signal

Table 1: SNR Enhancement table

\begin{tabular}{|c|c|c|c|}
\hline $\begin{array}{c}\text { Signal } \\
\text { identifi } \\
\text {-cation }\end{array}$ & $\begin{array}{c}\text { SNR } \\
\text { Original }\end{array}$ & $\begin{array}{c}\text { SNR } \\
\text { Enhancement } \\
\text { by classic } \\
\text { approach of } \\
\text { filtering }\end{array}$ & $\begin{array}{c}\text { SNR } \\
\text { Enhancement } \\
\text { by SSP } \\
\text { technique }\end{array}$ \\
\hline LF1-C & 3.36 & 8.37 & 25.41 \\
\hline LF2-C & 3.25 & 9.26 & 24.7 \\
\hline LF3-C & 2.48 & 8.4 & 24.49 \\
\hline LF4-C & 2.13 & 8.9 & 27.09 \\
\hline LF5-C & 1.53 & 8.25 & 27.9 \\
\hline LF6-C & 0.87 & 7.61 & 31.57 \\
\hline LF7-C & 0.63 & 7.65 & 27.38 \\
\hline LF8-C & 1.27 & 9.23 & 24.18 \\
\hline \multirow[t]{2}{*}{ LF9-C } & 0.23 & 7.62 & 27.5 \\
\hline & \multicolumn{3}{|c|}{$\begin{array}{l}\text { Average of original SNR }=1.75 \\
\text { Average of enhanced SNR by classic } \\
\text { approach }=8.3 \\
\text { Average of enhanced SNR by SSP technique } \\
=26.69\end{array}$} \\
\hline
\end{tabular}

\section{Conclusion}

In signal processing technique, time-frequency representation is the best procedure [5]. SSP is one of the techniques which will give information about the frequency component present in the signal and time representation of the signal[7]. Applying 
conventional filtering approach to non-stationary non-linear ultrasonic TOFD signals, the grainy noise cannot be removed fully due to scattering of microstructure material. Whilst SSP technique is already used in pulse-echo method, we identify SSP as a powerful technique in ultrasonic TOFD welding defect signals. SSP processed signals are having high signal to noise ratio than the classical filtering approach.

\section{Scope for future}

If the signals are processed we can easily identify defect location. If we able to identify the back-wall echo, we can clearly identify multiple flaws present in the signal. Using these techniques we can identify the quality of welding effectively and also we can avoid the structural accidents caused in nuclear power plant components and other industries.

\section{Acknowledgements}

We would like to thank Dr.B.Venkatraman, Head of Quality Assurance Division, Indira Gandhi Centre for Atomic Research (IGCAR), Kalpakkam for extending his valuable support for this project. We are also thankful to IGCAR for the financial support in accomplishing the project goals along with the welding defect TOFD signals

\section{REFERENCES}

[1] J.Kim, L.udpa, S.Udpa.2001.Multistage adaptive noise cancellation for ultrasonic NDE; NDT\&E international; 34; 319-328.

[2] Bilgutay N.M, Bencharit U, Saniie J. 2002. Enhanced ultrasonic imaging with split-spectrum processing and polarity thresholding. Acoustics, Speech and Signal Processing, IEEE Transactions; 37(10); 1590 - 1592.
[3] I. C. da Silva, M. H. S. Siqueira,D.P.M. Oliveira,R. R. da Silva, A. A. de Carvalho, J. M. A. Rebello.2002. Automatic Inspection using the TOFD Technique and Neural Networks; 8 ECNDT Barcelona.

[4] A. Lhe'mery, P. Calmon, I. Lecoeur-Tai"bi, R. Raillon, L. Paradis.2000.Modeling tools for ultrasonic inspection of welds; NDT\&E International 33. 499-513.

[5] Miguel Angel Rodrı'guez-Herna'ndez.2003.Ultrasonic nondestructive evaluation with spatial combination of WignerVille Transforms; NDT\&E International 36. 441-445

[6] Time of flight diffraction, ultrasonic testing, website: www.nde.com/tofd.htm

[7] Philippe Rubbers, Charles John Pritchard. Aug 2003. An overview of Split Spectrum Processing; NDT.net, 8(8); www.ndt.net/article/v08n08/rubbers/rubbers.htm

[8] DRAI Redouane, Khelil Mohamed \& Benchaala Amar. Flaw Detection in Ultrasonics Using Wavelets Transform and Split Spectrum Processing. www.ndt.net/article/wcndt00/papers/idn589/idn589.htm

[9] G Baskaran, C Lakshmana Rao and K Balasubramaniam. November 2007. Simulation of the TOFD technique using the finite element method, Insight 49(11).

[10] . Wladyslaw michnowski, Rafal MIS, Jaroslaw mierzwa, Grzegorz Latarowski zbm ultra. 25-28 Oct 2008. Ultrasonic Examination of Difficult Welds, 17th World Conference on Nondestructive Testing, Shanghai, China

[11] Eurico J. D'Sa K. R. Ramakrishnan, P. Krause, Defect Detection In Ultrasonic Images, CH2766-4/89/0000 0577,IEEE. 